\title{
Integrierte Versorgung - zwischen Anspruch, Wirklichkeit und Evidenz in der Forschung
}

KIRSTEN HOEPER, VOLKER ERIC AMELUNG, JUSTYNA HARTMANN, CHRISTIAN KRAUTH

Kirsten Hoeper, Institut für Epidemiologie, Sozialmedizin und Gesundheitssystemforschung, Medizinische Hochschule Hannover

Prof. Dr. Volker E. Amelung, Vorstandsvositzender, Bundesverband Managed Care e. V., Institut für Epidemiologie, Sozialmedizin und Gesundheitssystemforschung, Medizinische Hochschule Hannover

Justyna Hartmann, Institut für Epidemiologie, Sozialmedizin und Gesundheitssystemforschung, Medizinische Hochschule Hannover

PD Dr. Christian Krauth, Institut für Epidemiologie, Sozialmedizin und Gesundheitssystemforschung, Medizinische Hochschule Hannover

Chronische Erkrankungen, Multimorbidität und demografischer Wandel sind die Schlagworte, die oft im Zusammenhang mit den zukünftigen Herausforderungen des Gesundheitssystems genannt werden. Das fragmentierte deutsche Gesundheitssystem ist allerdings nicht in der Lage, diesen Anforderungen gerecht zu werden. Das Potenzial einer Integrierten Versorgung ist riesig, aber neue Versorgungsstrukturen haben sich bislang nicht durchsetzen können. Dieser Beitrag geht der Frage nach, in wie weit die aktuelle Evidenzlage zur Wirksamkeit von Integrierter Versorgung eine Richtung für die Entwicklung zukünftiger Versorgungsprogramme anweisen kann.

\section{Einleitung}

Das deutsche Gesundheitssystem wird im internationalen Vergleich als ein System mit hoher Qualität in der Versorgungsleistung eingestuft (Björnberg, EHI 2012). Der Leistungskatalog für die Versicherten ist umfassend und mit einem Zugang, der weitgehend unabhängig vom Einkommen ist. Es besteht eine beinahe Vollversicherung für die gesamte Bevölkerung mit hohen Qualitätsstandards (Amelung, Hildebrandt et al. 2012). Die Gesundheitsausgaben sind im internationalen Vergleich allerdings hoch (OECD 2011).

Eine zunehmende Multimorbidität und die wachsende Bedeutung chronischer Erkrankungen stehen im Mittelpunkt zukünftiger Versorgungsherausforderungen. Im Rahmen der demografischen Entwicklung und des medizinisch-technologischen Fortschrittes wird die Anzahl chronisch kranker Patienten vermutlich weiter ansteigen. Ende der 90er Jahre hatten ca. 43\% der Bevölkerung mindestens eine chronische Erkrankung und bei den über 65-jähringen betrug die Anzahl sogar $70 \%$. In der hausärztlichen Versorgung betreffen $80 \%$ aller Beratungen chronische Erkrankungen. Der Anteil chronischer Erkrankungen lag im Jahr 2000 bei $46 \%$ aller Erkrankungen und wird vermutlich bis 2020 auf $60 \%$ ansteigen (Gensichen, Muth et al. 2006).

Die Gesundheitsausgaben sind unter den Versicherten ungleich verteilt. Nach Daten der AOK Niedersachsen, (Krankenhaus-, Krankengeld- und Arzneimittelausgaben, ca. $60 \%$ der Gesamtausgaben), ergibt sich, dass auf $10 \%$ der Versicherten $80 \%$ der Ausgaben fallen (SVR KAG 2003). Grobe et al. (2003) fanden in einer empirische Analyse zur Charakterisierung von Hochnutzern im Gesundheitswesen anhand von Daten der Gmünder Ersatzkasse, dass auf $20 \%$ der Versicherten hier sogar 91,5\% der »zuordnungsfähigen Leistungsausgaben« entfallen. Dabei geht es hier nicht um seltene, teure Erkrankungen, 
sondern vielmehr um klassische Volkskrankheiten wie z.B. Herzkreislauferkrankungen, psychische Erkrankungen, oder Diabetes Mellitus. Ein Großteil der Ausgaben entfällt somit auf einen relativ kleinen Teil der Versicherten. Daraus ergibt sich die Konsequenz, die Qualität und die Kosten der gesundheitlichen Versorgung dieser Versichertengruppe gezielt zu verbessern. Die Qualität eines Gesundheitssystems zeichnet sich insbesondere durch die Versorgung von chronisch kranken Patienten mit komplexen Krankheitsbildern aus (Amelung 2012a).

Durch die Fragmentierung des Versorgungssystems können sich jedoch Versorgungsdefizite ergeben, insbesondere an den Versorgungsschnittstellen. Schnittstellen existieren nicht nur zwischen ambulanter und stationärer Versorgung, sondern auch innerhalb des ambulanten Sektors. Hausärztliche, fach- und spezialfachärztliche Versorgung, Pflege und Rehabilitation werden jeweils von unterschiedlichen Leistungserbringern angeboten (SVR 2012). Die Defizite in der Behandlung chronisch kranker Patienten resultieren aus den Folgen der Fragmentierung bei der Versorgung über mehrere Sektoren hinweg, u. a. durch fehlende durchgehende Steuerung und Koordination der Versorgungsprozesse, limitierte Kommunikation zwischen den verschiedenen Leistungserbringern mit fehlender oder verzögerter Übermittlung von Patientendaten und fehlende sektorenübergreifende Therapieplanung mit der Gefahr von Versorgungsbrüchen und den Kosten durch Doppel- und Mehrfachuntersuchungen.

Dabei ist die Thematik alles andere als neu. Bereits 1975 hielt Jahn fest, dass »das System der medizinischen Versorgung in der Bundesrepublik Deutschland ... davon bestimmt (ist), daß

- an jeder Stelle, an der ein Versicherter in Behandlung tritt, ein diagnostischer Prozeß neu aufgenommen wird, ohne dass zuvor gewonnene Untersuchungsergebnisse einbezogen werden

- der Informationsaustausch zwischen den Ärzten minimale Ausmaße hat

- eine Kontinuität der Behandlung beim Wechsel der Versicherten zwi- schen den Subsystemen nicht zustande kommt und auch nicht zustande kommen kann ...«(Jahn 1975, S. 42)

Schon seit längerer Zeit und vielfach diskutiert werden integrierte Versorgungsstrukturen als Bestandteil eines zukunftsweisenden Gesundheitssystems betrachtet. Mittels integrierter Versorgung soll eine bessere Verzahnung der Leistungsbereiche erreicht werden, insbesondere in Hinblick auf Qualitätsverbesserung und Kostenreduktion bei der Zielgruppe der Patienten mit chronischen Erkrankungen und Multimorbidität. Wesentliche Elemente sind hierbei u. a. eine Auflösung der traditionellen Grenzen zwischen den Versorgungsbereichen (Arztpraxis, Krankenhaus, Einrichtungen der Pflege und Rehabilitation) mit einer Koordination der Gesundheitsversorgung über den gesamten Verlauf der Erkrankung hinweg und der Einsatz strukturierter Informationsund Kommunikationsinstrumente, z. B. in Form von elektronischen Patientenakten.

Doch die Umsetzung tritt gewissermaßen auf der Stelle und einen nennenswerten Anteil an der Gesamtversorgung haben neue Strukturen bislang nicht erreichen können. Der Anteil der Krankenkassenausgaben für innovative Versorgungsformen an den Gesamtausgaben liegt durchschnittlich bei unter einem Prozent (Gersch 2011). Eine Verbesserung der Versorgungssituation bei der Behandlung von Patienten mit chronischen Erkrankungen in Kombination mit Multimorbidität wird langfristig nur umsetzbar sein, wenn die im Kern

\section{Die Umsetzung der Integrierten} Versorgung tritt auf der Stelle, ein nennenswerter Anteil an der Gesamtversorgung ist bislang nicht erreicht worden.

des Problems liegenden Probleme überwunden werden. Die derzeitige Situation mit einer Trennung der Sektoren und Berufsgruppen, und voneinander "abgeschotteten « Einzelpraxen wird den Anforderungen jedoch nicht gerecht und ist mit der Bewältigung dieser Herausforderungen perspektivisch überfordert
(Amelung 2012a).

In den vergangenen 15 Jahren hat der Gesetzgeber eine Reihe von Möglichkeiten geschaffen, neue Versorgungskonzepte umzusetzen: mehr Wettbewerb zwischen den Krankenkassen, mehr Wahlmöglichkeiten für die Versicherten und Patienten und mehr unternehmerischer Spielraum für die Akteure in den unterschiedlichen Sektoren des Gesundheitswesens (Amelung 2008). Durch das Gesundheitsmodernisierungsgesetz 2004 ist es möglich, dass einzelne Ärzte und Ärztenetze direkt Vertragspartner der Krankenkassen werden. Innerhalb von vier Jahren wurden ca. 6000 Verträge der Integrierten Versorgung abgeschlossen. Mit dem Auslaufen der Anschubfinanzierung Ende 2008 ging zunächst die Zahl zurück, doch durch eine Reihe von neuen Verträgen, z. B. im Bereich der psychischen Erkrankungen oder Rheumatologie liegt die Anzahl der Verträge nun bei ungefähr 6300 (SVR 2012).

Der Bedarf einer integrierten Versorgung bei einer steigenden Zahl von Patienten mit chronischen kostenintensiven Erkrankungen ist unter Experten unbestritten. Darüber hinaus hat der Gesetzgeber in den vergangenen Jahren Rahmenbedingungen geschaffen, neue Versorgungskonzepte umzusetzen. Das Konzept der Integrierten Versorgung ist überzeugend, dennoch fehlt nach wie vor der Durchbruch in Deutschland.

\section{Systematische Literatursuche}

\subsection{Ziele}

Im Rahmen eines EU-Projektes »International Research on Financing Quality in Healthcare - Interquality ${ }^{1}$ wurde eine systematische Literaturrecherche zum Thema "Integrierte Versorgung " durchgeführt. Hierbei ging es insbesondere darum, die aktuelle Evidenzlage im Bereich der Integrierten Versorgung in Bezug auf Wirksamkeit und Kosteneffektivität herauszuarbeiten.

Das Verständnis von integrierter Versorgung ist ausgesprochen heterogen, nicht nur weltweit, sondern auch innerhalb Europas. Dabei müssen jeweils die verschiedenen nationalen Rahmenbedingungen, Finanzierungs-

\footnotetext{
1 Framework 7

http://www.interqualityproject.eu/
} 
systeme, grundsätzlichen sozialen Werte und politischen Ansätze berücksichtigt werden. Bislang gibt es keine einheitliche Definition oder gar einen einheitlichen Begriff, sondern eher eine Fülle von verschiedenen Definitionsansätzen. Es werden oft die Ausdrücke »chronic care programmes, shared care, chronic care model und disease management programme" mit synonymer Bedeutung genutzt. In Deutschland wird die Integrierte Versorgung als »eine verschiedene Leistungssektoren übergreifende Versorgung der Versicherten oder eine interdisziplinär-fachübergreifende Versorgung « (Legaldefinition $\mathbb{S} 140 \mathrm{a}$ SGB V) beschrieben. Eine exakte Definition der Integrierten Versorgung ist im Gesetz nicht verankert und der Gesetz-

\section{Das Verständnis von Integrierter Versorgung ist innerhalb Europas und in anderen Ländern ausgesprochen heterogen.}

geber überlässt die Wahl der Integrationstiefe sowie die Intensität der Kooperationsbeziehungen weitestgehend den Vertragspartnern (Mühlbacher, Lubs et al. 2006).

Neben einer fehlenden einheitlichen Definition von Integrierter Versorgung gibt es bislang wenig einheitliche Evidenz für eine Steigerung der Qualität der Behandlung, gemessen an klinischen Endpunkten, Kosteneffektivität und der Zufriedenheit von Leistungserbringern und -empfängern. Auf der Basis von systematischen Reviews und Meta-Analysen soll in dieser Arbeit Qualität und Umfang der Effekte von integrierter Versorgung bei Patienten mit chronischen Erkrankungen untersucht werden.

Die Forschungsfrage fokussierte auf Evidenz in Bezug auf 1. verbesserte Versorgungsqualität (klinische Endpunkte), 2. Kosteneffektivität und 3. patientenbezogene Ergebnisse wie Lebensqualität und Patientenzufriedenheit. Ziel dieses Reviews ist die Zusammenfassung und Bewertung der aktuellen Forschungsergebnisse zur Effektivität von Integrierter Versorgung von Patienten mit chronischen Erkrankungen in Europa, gemessen an der Qualität der Versorgung anhand von klinischen Endpunkten und gesundheitsökonomischer Betrachtung

Tab. 1: Anzahl der eingeschlossenen Primärstudien pro Indikation

\begin{tabular}{|llll|}
\hline Indikation & $\begin{array}{l}\text { Primärstudien } \\
\text { berichtet } \\
\text { Gesamt/Europa }\end{array}$ & $\begin{array}{l}\text { Duplikate } \\
\text { Gesamt/Europa }\end{array}$ & $\begin{array}{l}\text { Primärstudien } \\
\text { Gesamt/Europa }\end{array}$ \\
\hline CHF & $59 / 30$ & $6 / 5$ & $53 / 25$ \\
COPD & $54 / 29$ & $16 / 8$ & $38 / 21$ \\
Diabetes* $_{\text {Krebserkrankungen* }}^{*}$ & $78 / 43$ & $6 / 4$ & $72 / 39$ \\
$\begin{array}{l}\text { Psychische } \\
\text { Erkrankungen* }\end{array}$ & $31 / 18$ & $0 / 0$ & $31 / 18$ \\
Andere* & $9 / 3$ & $0 / 0$ & $9 / 3$ \\
Gesamt & $7 / 6$ & $0 / 0$ & $7 / 6$ \\
\hline *in dieser Arbeit nicht eingeschlossen & $\mathbf{2 8 / 1 5}$ & $\mathbf{2 1 0 / 1 1 2}$ \\
\hline
\end{tabular}

in klinischen Studien und patientenbezogene Endpunkte wie Lebensqualität und Patientenzufriedenheit.

\subsection{Methoden}

Zur Identifikation von systematischen Reviews und MetaAnalysen zur Effektivität von Versorgungsprogrammen in Europa wurde eine systematische Literaturrecherche durchgeführt.

Eingeschlossen wurden Systematische Reviews und Meta-Analysen aus den Jahren 2002 bis 2012 in englischer oder deutscher Sprache mit einer explizit definierten Forschungsfrage. Die eingeschlossenen Primärstudien sollten zum überwiegenden Teil aus Europa stammen und über ein experimentelles Design verfügen wie z. B. randomisierte klinische Studien (RCT) mit einem Anteil von mindestens 50\% RCT der eingeschlossenen Primärstudien. Studienteilnehmer waren Erwachsene mit chronischen Erkrankungen, die an einem integrierten Versorgungsprogramm teilgenommen haben. Die Ergebnisparameter wurden durch die Forschungsfragen der für die Reviews oder Meta-Analysen ausgewählten Studien bestimmt. Systematische Reviews oder Meta-Analysen wurden ausgeschlossen, wenn die Primärstudien keine Prozessoder Programmergebnisse gemessen oder ausschließlich diagnostische Prozeduren, pharmakologische Substanzen, Patientenschulungsprogramme oder krankenhausinterne Prozesse untersucht haben. Es wurde eine Recherche in Medline, Scopus, Embase, Wholis und Cochrane Library of Systematic Reviews durchgeführt, unter Verwendung folgender Suchbegriffe: disease management, chronic care, coordinated care, chronic disease management, integrated care, integrated services, and disease management program. Die Suche wurde kombiniert mit den Suchbegriffen: quality*, quality improvement, effectiveness, cost-effectiveness, und cost*. Zusätzlich wurde in Literaturverzeichnissen von Reviews recherchiert.

\section{Ergebnisse \\ 2.1 Übersicht}

Durch Kombination der Suchbegriffe verblieben nach anfänglich 25.135 Treffern noch 889 Artikel zur weiteren Durchsicht. Nach Ausschluss von Duplikaten und Screening des Titels verblieben 409 Abstracts zur weiteren Analyse. Davon erfüllten 101 Abstracts die Selektionskriterien. Durch die Handsuche wurden 9 weitere Artikel identifiziert. Letztendlich wurden jedoch 100 Artikel u. a. aufgrund methodischer Mängel, Art der Publikation oder geografischer Verteilung ausgeschlossen.

Insgesamt wurden zehn systematische Reviews identifiziert, vier davon mit zusätzlicher Meta-Analyse (Inglis 2010, Lambrinou 2012, Lemmens 2011, Peytremann-Bridevaux 2008).

Von den 238 eingeschlossenen Primärstudien stammten 129 aus Europa:

$38 \%$ aus UK ( $\mathrm{n}=43), 26 \%$ aus den Niederlanden $(n=30), 14 \%$ aus Skandinavien (Schweden $n=8$, Dänemark $n=5$, Norwegen $n=2$, Finnland $n=1) ; 6 \%$ aus Spanien $(\mathrm{n}=7), 4,5 \%$ aus Irland $(\mathrm{n}=5)$ und Deutschland $(n=5)$ und $1 \%$ aus Frankreich $(\mathrm{n}=1)$.

Es wurden verschiedene chronische Erkrankungen untersucht. Drei Reviews fokussierten jeweils auf chroni- 
Tab. 2: Programmbezeichnungen und Programmkomponenten

\begin{tabular}{|c|c|}
\hline Autor/Programmbezeichnung & Programme \\
\hline \multicolumn{2}{|l|}{ Chronische Herzinsuffizienz } \\
\hline Inglis 2011 & Strukturierte telefonische Unterstützung: \\
\hline $\begin{array}{l}\text { Disease Management } \\
\text { Programme }\end{array}$ & $\begin{array}{l}\text { - Monitoring und/oder Management zur Selbstversorgung wird über einfache } \\
\text { Telefontechnologie realisiert. Daten werden mit Hilfe eines Computers gesammelt und } \\
\text { gespeichert } \\
\text { Telemonitoring: } \\
\text { - Übertragung von physiologischen oder anderen nicht invasiven Daten }\end{array}$ \\
\hline $\begin{array}{l}\text { Lambrinou } 2012 \\
\text { Nurse oriented Heart Failure } \\
\text { Management Programme }\end{array}$ & $\begin{array}{l}\text { Unterstützung vor Krankenhausentlassung } \\
\text { - Entlassungsplanung durch Pflegepersonal } \\
\text { Unterstützung nach Krankenhausentlassung } \\
\text { - Stärkung der Selbstversorgung und kontinuierliche Schulung }\end{array}$ \\
\hline $\begin{array}{l}\text { Phillips } 2005 \\
\text { Nurse oriented Heart Failure } \\
\text { Disease Management }\end{array}$ & $\begin{array}{ll}\text { - } & \text { Entlassungsplanung } \\
\text { - } & \text { Nachsorge in einer pflege-geleiteten CHF Klinik } \\
\text { - } & \text { Management der Selbstversorgung, Telefonkontakt } \\
\text { - } & \text { CHF Schulung nach Entlassung (Patienten und Angehörige), Hausbesuche } \\
\end{array}$ \\
\hline \multicolumn{2}{|r|}{ I } \\
\hline $\begin{array}{l}\text { Lemmens } 2011 \\
\text { Chronic Care Management }\end{array}$ & $\begin{array}{ll}\text { - } & \text { Selbstmanagement z.B. Schulung oder Zieldefinitionen } \\
\text { - } & \text { Versorgungssystem (regelmäßige Follow-ups, andere Team Rollen) } \\
\text { - } & \text { Entscheidungsunterstützung (z.B. Integration evidenzbasierter klinischer Leitlinien durch } \\
& \text { Erinnerungssysteme oder Feedback System } \\
\text { - } & \text { Klinische Informationssysteme (z.B. Informationssysteme mit Erinnerungs-, Feedback- } \\
& \text { oder Leistungsunterstützung }\end{array}$ \\
\hline $\begin{array}{l}\text { Peytremann-Bridevaux } 2008 \\
\text { Disease Management } \\
\text { Programme }\end{array}$ & $\begin{array}{l}\text { - } \quad \text { zwei oder mehr unterschiedliche Komponenten (z.B. Bewegungsübungen, } \\
\text { Selbstmanagement, strukturiertes Follow-up) } \\
\text { - } \quad \text { zwei oder mehr Akteure aktiv in Patientenversorgung involviert } \\
\text { - Patientenschulung } \\
\text { - Mindestens eine Komponente der Intervention dauert über mindestens ein Jahr }\end{array}$ \\
\hline $\begin{array}{l}\text { Niesink } 2007 \\
\text { Chronic Disease Management }\end{array}$ & $\begin{array}{l}\text { - } \text { Schulung, Übungen } \\
\text { - Versorgungsplan, Aktionsplan } \\
\text { - Psychosoziale Unterstützung, Entspannungstechniken } \\
\text { - Tabakentwöhnung } \\
\text { - Atemtraining }\end{array}$ \\
\hline
\end{tabular}

sche Herzinsuffizienz (CHF) (Inglis 2011, Lambrinou 2012, Phillips 2005) und chronisch-obstruktiver Lungenerkrankung (COPD) (Lemmens 2011, Peytrmann-Bridevaux 2008, Niesink 2007). Zwei Reviews konzentrierten sich auf Diabetes (Seitz 2011, Dorrestejn 2010) und einer auf Krebserkrankungen (Ouwens 2009). Das von Smith (2007) durchführte Review legte den Schwerpunkt auf chronische Erkrankungen im Allgemeinen und hat Studien mit unterschiedlichen Indikationen eingeschlossen (Diabetes, Asthma, CHF, Krebserkrankungen, Hypertonus, COPD, Depression). 87\% der eingeschlossenen Primärstudien waren randomisierte klinische Studien. Im weiteren Verlauf werden nur die Reviews mit den Indikationen CHF und COPD betrachtet.

Die Definitionen von Integrierter Versorgung und die einzelnen Programme variierten ganz erheblich in den eingeschlossenen Artikeln (Tabelle 2). Der Begriff »Integrierte Versorgung « wird in den USA und Europa unterschiedlich verwendet. In den USA liegt der Schwerpunkt auf Gesundheitsleistungen, die durch private und staatliche Versicherungen abgedeckt sind. In Europa umfasst integrierte Versorgung häufig auch Leistungen außerhalb des Gesundheitssystems, z. B. in Altenheimen oder Sozialdienste wie Gemeindeschwestern. Auf einen gemeinsamen Nenner heruntergebrochen ergeben sich aus den eingeschlossenen Reviews folgende Kriterien:

- die Intervention oder das Programm soll aus zwei oder mehr verschiedenen Komponenten bestehen (Peytremann-Bridevaux, Staeger et al. 2008; Dorresteijn, Kriegsman et al. 2010; Lemmens, Lemmens et al. 2011)
- und/oder verschiedene Sektoren einschließen, z. B. multidisziplinäre Versorgungsteams (Niesink, Trappenburg et al. 2007; Ouwens, Hulscher et al. 2009; Lambrinou, Kalogirou et al. 2012).

\subsection{Effektivität von Integrierter Versorgung}

Die Programme der Integrierten Versorgung der eingeschlossenen Reviews unterscheiden sich u. a. ganz erheblich in ihrer Programmstruktur, Programmdauer, Zielgruppe und beteiligten Akteuren des Gesundheitswesens. Gemeinsame Aspekte der Programme sind der starke Fokus auf die Verringerung der Mortalität, eine Verbesserung des Gesundheitsstatus, die Verbesserung der Lebensqualität und die Reduktion von 
Krankenhauseinweisungen. Die Ergebnisparameter der eingeschlossenen Reviews variieren, aber häufig gemessene Variablen waren, in Abhängigkeit von der untersuchten Indikation:

- Lebensqualität

- Krankenhauseinweisungen

- Mortalität

- Morbidität

Die Ermittlung der Lebensqualität und Endpunkte in Bezug auf Krankenhauseinweisungen waren die am häufigsten ermittelten Parameter in fünf von sechs Reviews. Mortalität als Endpunktparameter wurde in vier Reviews beschrieben, Morbidität nur in einem Review. In zwei Reviews wurden zumindest einige Informationen zu Kosten, Kosteneffektivität oder Kosteneinsparungen gegeben (Inglis 2010, Phillips 2005). Aufgrund der Heterogenität der Erkrankungen, Programme und gemessenen Endpunkte konnte keine Synthese der Studienergebnisse durchgeführt werden.

\section{Lebensqualität}

Alle Reviews, die den Einfluss von integrierter Versorgung bei Patienten mit COPD untersucht haben, haben die Lebensqualität bewertet. Lemmens (2007) zeigt, dass gepoolte Ergebnisse keine signifikante Verbesserung bringen. Allerdings zeigen Studien mit guter methodischer Qualität eine signifikante Verbesserung der Lebensqualität. Im Review von Peytrmann-Bridevaux (2008) kann in sieben von zwölf eingeschlossenen Studien eine signifikante Verbesserung der Lebensqualität gezeigt werden. Niesink (2007) berichtet ebenfalls in sieben (von zehn) Studien von signifikanten klinisch relevanten Unterschieden in der Lebensqualität. Allerdings überschneiden sich vier eingeschlossene Primärstudien mit den anderen Reviews.

Die Ergebnisse in Bezug auf Lebensqualität bei chronischem Herzversagen waren sehr gemischt, nur wenige Primärstudien zeigten signifikante Verbesserungen.

\section{Krankenhauseinweisungen}

Krankenhauseinweisungen aller Ursachen und Einweisungen durch CHF begründet wurden in allen drei Reviews, die diese Indikation betreffen bewertet. Entlassmanagement erscheint eine vielversprechende Strategie zu sein, beide Formen der Krankenhauseinweisungen zu reduzieren. Phillips (2005) berichtet von der Reduktion des relativen Risikos von $70 \%$ für eine erste Wiedereinweisung und einer ebenfalls 70prozentigen Reduktion des relativen Risikos einer krankheitsspezifischen Wiedereinweisung im Vergleich zur Standardversorgung. Leider liegen diesem Resultat nur zwei Primärstudien mit 218 Patienten zugrunde. Lambrinou (2012) zeigt ebenfalls eine signifikante Reduktion der stationären Aufnahme. In einem Review

\section{Nur wenige Primärstudien haben den Effekt von Integrierter Versorgung auf die Kosten untersucht.}

(Inglis 2010) wurden strukturierte Telefonunterstützung (STS) und Telemonitoring Maßnahmen (TM) untersucht. Beide Interventionen zeigen eine signifikante Risikoreduktion bei Krankenhauseinweisung aller Ursachen und auch bei CHF-induzierten Aufnahmen in ein Krankenhaus.

Zwei Reviews mit Fokus auf COPD berichten von positiven Effekten von Interventionen auf Krankenhauseinweisungen. Peytrmann-Bridevaux (2008) zeigt einen signifikanten Rückgang des Risikos von Einweisungen in sieben von zehn Primärstudien, während die gepoolten Ergebnisse von Lemmens (2011) keine signifikante Verbesserung zeigen.

\section{Mortalität}

Die Wirkung von Integrierter Versorgung auf Mortalität wurde in vier Reviews untersucht. Für Patienten mit COPD gab es keinen positiven Effekt (Lemmens 2011, Peytrmann-Bridevaux 2008). In zwei Reviews mit CHF Patienten blieben die Ergebnisse uneindeutig. Ein Review (Inglis 2010) zeigt, dass Telemonitoring signifikant effektiv war mit einer Reduktion der Mortalität von $34 \%$. Ein ähnlicher, allerdings nicht signifikanter Trend wurde für die strukturierte Telefonunterstützung gefunden.

\section{Morbidität}

Nur in einem der drei Reviews mit Fokus auf COPD wurde die Wirkung von integrierter Versorgung auf die körperliche Belastbarkeit und Lungenfunktion untersucht (Peytrmann-Bridevaux 2008). Lungenfunktion und Belastbarkeit (Gehtest) wurden in sieben Primärstudien bewertet. Drei Studien fanden eine signifikante Verbesserung der Gehstrecke, aber eine von ihnen wurde als möglicherweise klinisch nicht relevant eingestuft. Nur eine Primärstudie wies Evidenz für eine signifikante Verbesserung der Lungenfunktion nach.

\section{Kosten}

Nur zwei der sechs Reviews beschreiben die Wirkung der Programme auf Kosten in Form von Kosteneinsparungen oder Gesamtausgaben, beide bezogen auf CHF (Inglis 2010, Phillips 2005). Zwölf Primärstudien aus dem Review von Inglis (2010) beschreiben Details zu den Kosten der Interventionen, Kostenreduktionen oder Kosteneffektivität. Acht Studien zeigen Kostensenkungen mit Einsparungen zwischen $14 \%$ und $86 \%$.

Phillips 2005 fand zusätzliche Initialkosten, die mit der Administration der Intervention verbunden waren von US\$ 74.50 pro Patient und Monat und potenzielle Einsparungen von US\$277,88 pro Patient und Monat (US\$108.25 555.67) bei den komplexeren Programmen (Primärstudien zu weniger komplexen Programmen enthielten keine Informationen zu den Kosten.)

\section{Diskussion}

\subsection{Ergebnisse}

Es wurden zehn Systematische Reviews und Meta-Analysen mit Schwerpunkt Europa identifiziert, die zwischen 2005 und 2012 publiziert wurden und sich auf verschiedene chronische Erkrankungen und unterschiedliche Konzepte von Integrierter Versorgung konzentrierten.

Der Effekt von Integrierter Versorgung bei chronischen Erkrankungen wurde zumeist für die Lebensqualität untersucht. Ergebnisse der Reviews, welche die Wirksamkeit bei Patienten mit COPD untersucht haben legen nahe, dass Integrierte Versorgung die 
Tab. 3: Erfolgsfaktoren

\begin{tabular}{|c|c|}
\hline Autor & Erfolgsfaktoren \\
\hline \multicolumn{2}{|l|}{$\mathrm{CHF}$} \\
\hline Inglis 2011 & $\begin{array}{l}\text { - Unterstützung des Selbstmanagements und »Remote } \\
\text { Management« }\end{array}$ \\
\hline $\begin{array}{l}\text { Lambrinou } \\
2012\end{array}$ & - Entlassmanagement \\
\hline Phillips,2005 & $\begin{array}{l}\text { - Entlassmanagement } \\
\text { - Frühzeitiges Follow-up nach Krankenhausentlassung }\end{array}$ \\
\hline \multicolumn{2}{|l|}{ COPD } \\
\hline Lemmens 2011 & $\begin{array}{l}\text { - Kombination mehrerer Interventionselemente } \\
\text { - Populationsbasierter Ansatz }\end{array}$ \\
\hline Niesink 2007 & $\begin{array}{l}\text { - Integration hausärztlicher Versorgung } \\
\text { - Qualität der Kommunikation und Zusammenarbeit zwischen } \\
\text { - Keistungserbringern } \\
\text { - Kommunikation mit Patienten }\end{array}$ \\
\hline
\end{tabular}

Lebensqualität verbessern könnte. Einen signifikanten Hinweis auf eine Verbesserung der Lebensqualität bei Patienten mit chronischer Herzinsuffizienz gibt es hingegen nicht. Allerdings darf insgesamt dabei nicht übersehen werden, dass bislang keine einheitlichen und damit direkt vergleichbaren Erhebungsinstrumente verwendet werden. Entlassmanagement als qualitatives Element der Patientenversorgung bei Patienten mit Herzinsuffizienz scheint eine vielversprechende Strategie zu sein, um sowohl Einweisungen für alle Ursachen, als auch krankheitsspezifische Krankenhauseinweisungen zu reduzieren. Eine gute Entlassungsplanung trägt darüber hinaus zur Vermeidung von Wiedereinweisungen bei. Es wurde keine Evidenz auf Verringerung der Mortalität bei Patienten mit COPD gefunden, die Ergebnisse für Patienten mit chronischer Herzinsuffizienz waren nicht eindeutig. Allerdings zeigte sich eine Verbesserung der körperlichen Belastbarkeit bei Patienten mit COPD. Eine gesundheitsökonomische Bedeutung kann in diesem Rahmen nicht diskutiert werden. Leider haben nur wenige Primärstudien den Effekt von integrierter Versorgung auf die Kosten untersucht. Trotz der wachsenden Bedeutung gesundheitsökonomischer Analysen gibt es offensichtlich noch immer eine ungenügende Einbindung entsprechender Parameter in klinische Studien.

Auch wenn vereinzelt statistisch signifikante Effekte gezeigt werden konnten, lässt sich eine indikationsübergreifende positive Wirkung dabei nicht festhalten.

\subsection{Erfolgsfaktoren}

Nach wie vor kann die Frage nach Erfolgsfaktoren nicht indikationsübergreifend umfassend und abschließend beantwortet werden. Zwei Reviews mit Schwerpunkt auf Herzinsuffizienz identifizieren »Entlassmanagement « und »frühes klinisches Follow-up«, als Erfolgsfaktoren. Phillips (2005) zeigt den Nutzen insbesondere für stabile Patienten mit guter Kontrolle der Symptome. Inglis (2011) zeigt, dass Telemonitoring möglicherweise nicht so effektiv bei stabilen Patienten ist, sondern eher eine effiziente und kostengünstige Option bei Patienten, deren Qualität der Versorgung nicht dem Standard der klinischen Studien entspricht. In diesem Review werden »Verbessertes Selbstmanagement « und »Remote Management « als potenzielle Schlüsselfaktoren der verbesserten Qualität der Versorgung identifiziert. Die Versorgungsprogramme der eingeschlossenen Studien von Lemmens (2011) wurden bei Patienten mit eher schwerer COPD untersucht. Die Autoren diskutieren, dass die Kombination mehrerer Komponenten und mit einem populationsbasierten Ansatz einen Einfluss auf die Qualität und Wirksamkeit der integrierten Versorgung haben könnte. Niesink (2007) betrachtete ausschließlich den Einfluss der integrierten Versorgung auf die Lebensqualität der Patienten mit COPD und betont in diesem Zusammenhang die Bedeutung der Qualität der Kommunikation und
Zusammenarbeit zwischen Leistungserbringern und Kommunikation mit den Patienten. Eine Integration der hausärztlichen Versorgung zusätzlich zur fachärztlichen Betreuung schien hier einen positiven Effekt zu haben (Tabelle 3).

\section{Schlussfolgerungen}

Auf der Grundlage einer ausgesprochen heterogenen Gruppe von Systematischen Reviews und Meta-Analysen lässt sich keine Schlussfolgerung zur Effektivität von Integrierter Versorgung über alle Indikationen hinweg ziehen. Die dargestellten Effekte der Integrierten Versorgung auf der Basis von randomisierten klinischen Studien mit Fokus auf Europa lassen sich zweifelsohne auch nicht direkt auf den Versorgungsalltag und das deutsche Gesundheitssystem übertragen.

Nichtsdestotrotz lässt sich anhand der Evidenzlage zumindest ein Hinweis auf eine mögliche Richtung der weiteren Entwicklung erkennen. Auch wenn sich auf der Basis dieser Untersuchung keine allgemeingültigen Schlussfolgerungen ziehen lassen, so stellt sich doch die Frage, ob strukturierte Behandlungsansätze indikationsunabhängig erarbeitet werden können. Die Bedürfnisse der

\section{Es stellt sich die Frage, ob strukturierte Behandlungs- ansätze indikationsunabhängig erarbeitet werden können.}

unterschiedlichen Patientengruppen scheinen doch sehr komplex und heterogen zu sein. Ein Erfolgsfaktor, wie hier in der Versorgung von Patienten mit COPD gezeigt, könnte sein, den individuellen Versorgungsbedarf von Patienten mit chronischen Erkrankungen mit unterschiedlichem Schweregrad der Erkrankung in Betracht zu ziehen. Auch in der Indikation Herzinsuffizienz hat sich gezeigt, dass Interventionen je nach Erkrankungsgrad unterschiedlich wirksam sind.

Erfolgsfaktoren für die Versorgung von Patienten mit Herzinsuffizienz können ein Entlassmanagement, frühzeitiges Follow-up nach Krankenhausentlassung, Telemonitoring und eine Unterstützung des Selbstmanagements 
sein. Hausärzte warten laut einer Umfrage allerdings bis zu 30 Tage und mehr auf einen Entlassungsbrief (The Commonwealth Fund 2006). Durch eine fehlende IT-Vernetzung und Standards der Datenweitergabe kommt es bei der Übermittlung von Patientendaten zu erheblichen Zeitverzögerungen, verbunden mit dem Risiko einer mangelhaften medizinischen Versorgung (Henze and Pöppl 2005). Ein Schritt in die richtige Richtung ist die im Rahmen der Einführung des Versorgungsstrukturgesetzes vorgesehene Verpflichtung der Krankenhäuser zu einem verbindlichen Entlassmanagement (Ergänzung $\int 39$ SGB V). Sollte es gelingen, u. a. einen zuverlässigen, zeitnahen Informationsfluss $\mathrm{zu}$ etablieren, würde die Phase der nachstationären Betreuung erheblich verbessert und Therapiebrüche könnten vermieden werden (Amelung and Wolf 2012).

Das Gesetz für die Integrierten Versorgungskonzepte schreibt keine unabhängige, verbindliche Evaluation mit einem entsprechenden Ergebnisbericht vor (SVR 2003, Nr. 700). Dadurch mangelt es erheblich an Transparenz und die Vorteile innovativer Versorgungsformen sind kaum erkennbar. Insbesondere $\mathrm{Pa}$ tienten können die unterschiedlichen Angebote der Krankenkassen kaum beurteilen. Auf der anderen Seite wird auch mit steigender Transparenz die Bedeutung der Integrierten Versorgung als Wettbewerbsinstrument für die Krankenkassen zunehmen. Deshalb ist es besonders wichtig, dass standardisierte Evaluationen für innovative Versorgungsformen durchgeführt und die Ergebnisse öffentlich zugängig gemacht werden (Amelung and Wolf 2012).

Eine der entscheidenden Fragen bei der Evaluation von integrierten Versorgungssystemen ist, welche Methodik sinnvoll angewendet werden kann. Im Rahmen dieser Arbeit wurde der Fokus auf randomisierte klinische Studien gelegt, welche als Goldstandard für die Qualität einer medizinischen Evaluation gelten. In diesen Studien werden Patienten, die von einer Intervention profitieren könnten, nach einem $\mathrm{Zu}$ fallsverfahren einer Interventionsgruppe und einer Kontrollgruppe zugeordnet und die Ergebnisse verglichen. Bei einer guten Randomisierung unterscheiden sich beide Gruppen nur bezüglich der Interventionsmerkmale (Amelung 2012a). Die Frage ist allerdings, ob eine
Evaluation komplexer sozialer Institutionen oder Phänomene und Verträge mit den im Medizinbetrieb üblichen Instrumentarium erfolgen kann oder Anpassung benötigt, weil sich der Alltag der Versorgung nicht über eine randomisierte klinische Studie abbilden lässt (Schmacke 2011). Ziel der Evaluation der integrierten Versorgung ist die Überprüfung in Bezug auf Wirtschaftlichkeit und Qualität z. B. im Vergleich zur Regelversorgung. Methodisch wichtige Aspekte an dieser Stelle sind die Bestimmung der Vergleichsgruppe in der Regelversorgung und die Bestimmung und Ermittlung von Qualitäts- und Kostenindikatoren. Verträge der integ-

\section{Es mangelt weiterhin an eindeutiger Evidenz, dass eine Integrierte Versorgung qualitativ besser und kostengünstiger ist.}

rierten Versorgungsprogramme können sich entweder auf einzelne Indikationen beziehen (indikationsbezogene integrierte Versorgung) oder können prinzipiell für alle Indikationen einer definierten Population gelten (populationsbezogene integrierte Versorgung oder auch integrierte Vollversorgung). Eine Analyse auf der Basis von RCT kann nur indikationsbasiert sein, denn auf der populationsbasierten Ebene ist eine Randomisierung schwierig. Problematisch ist auch die Identifikation einer Gruppe von Patienten in der Regelversorgung, die den Patienten in der integrierten Versorgung entsprechen, um einen Vergleich zu gewährleisten. Studien, in denen keine Randomisierung vorgesehen ist, haben ein hohes Risiko eines Selection Bias, da die Aufteilung nicht zufällig erfolgt, sondern durch Merkmale der Teilnehmer beeinflusst wird.

Die Daten unserer Arbeit zeigen, dass es weiterhin an eindeutiger Evidenz mangelt, dass eine Integrierte Versorgung qualitativ besser und kostengünstiger ist. Hier stellt sich allerdings die Frage, ob die Evidenz so nicht messbar oder nachweisbar ist, oder ob sie gänzlich fehlt. Die Qualität der einzelnen Programme in den eingeschlossenen Primärstudien wird nicht diskutiert und müsste mehr untersucht und beschrie- ben werden. So lässt sich nicht zweifelsfrei zuordnen, ob die fehlende Evidenz an einer nicht vorhandenen Wirksamkeit oder eher an der Qualität der Programme liegt. Dadurch, dass der Gesetzgeber keine unabhängige, verbindliche Evaluation fordert (s.o.), fehlen wissenschaftlich publizierte Ergebnisse zu bereits implementierten Programmen in Deutschland. Bisher sind IV-Verträge gar nicht oder nur unzureichend evaluiert worden. Hierbei handelt es sich jedoch zumeist um Geschäftsmodelle mit einem Wettbewerbsansatz als Zielrichtung. Auch wenn zu erwarten ist, dass zukünftig mehr Programme evaluiert werden, steht zu befürchten, dass die Ergebnisse wenig Akzeptanz finden aufgrund der bisher häufig ungenügenden Qualität der Evaluationen. Es stellt sich auch die Frage, welche Methode geeignet ist, Verträge der Integrierten Versorgung als Geschäftsmodell oder Unternehmenskonzept zu evaluieren. Umso mehr ist eine Standardisierung der Evaluation unvermeidbar. In einem nächsten Schritt sollte ein gesetzlicher Rahmen geschaffen werden, welcher die Bedingungen der Evaluation festlegt.

Bislang haben sich die Erwartungen an eine qualitativ bessere und kostengünstigere Versorgung durch neue Versorgungskonzepte nicht bestätigt und es fehlen nach wie vor empirische Daten zum Nachweis der Wirksamkeit. Die Integrierte Versorgung wird sich vermutlich erst durchsetzen, wenn der Nachweis einer Verbesserung der Versorgung im Vergleich zur Regelversorgung erbracht wird. Für weitere empirische Untersuchungen sollten Standards für die Evaluation entwickelt und ein institutioneller Rahmen geschaffen werden.

Dieses Projekt wird durch das Framework 7 Programm der Europäischen Union gefördert (FP7/2007-2012, Grant Agreement Nr. 261369). 


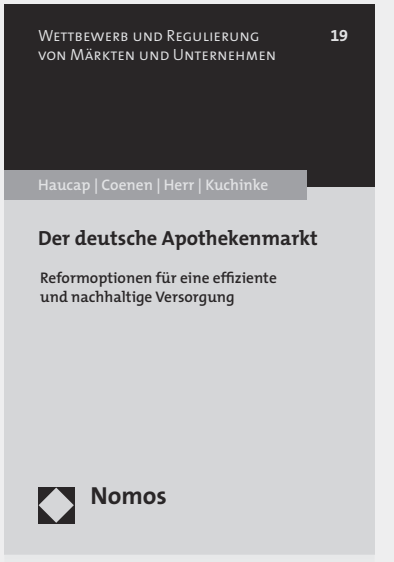

\section{Der deutsche Apothekenmarkt}

Reformoptionen für eine effiziente und nachhaltige Versorgung

Von Prof. Dr. Justus Haucap,

Dr. Michael Coenen,

Dr. Annika Herr,

PD Dr. Björn A. Kuchinke

2012, 94 S., brosch., 22,- $€$

ISBN 978-3-8329-7068-0

(Wettbewerb und Regu-

lierung von Märkten und

Unternehmen, Bd. 19)

Die Autoren analysieren den Ordnungsrahmen für den deutschen Apothekenmarkt und die dortigen Wettbewerbsdefizite. Vorgeschlagen wird das Modell einer Apothekentaxe, bei dem die Patienten die Leistung der Apotheke direkt vergüten. Das mögliche Einsparpotenzial pro Jahr wird auf bis zu 448 Mio. EUR geschätzt.

www.nomos-shop.de/14183 $\gamma$ Nomos

\section{Literatur}

Amelung, V., H. Hildebrandt, et al. (2012): "Integrated care in Germany - a stony but necessary road! « Int J Integr Care 12: e16

Amelung, V. E. (2008): Managed Care Modell der Zukunft? Integrierte Versorgung und Medizinische Versorgungszentren. V. E. Amelung, K. Meyer-Lutherloh, E. Schmidet al. Berlin, MWY: S. 1-34.

Amelung, V. E. (2012a): Managed Care: Neue Wege im Gesundheitsmanagement Wiesbaden, Gabler Verlag, 5. Aufl.

Amelung, V. E. and S. Wolf (2012): »Integrierte Versorgung - Vom Hoffnungsträger zum Ladenhüter der deutschen Gesundheitspolitik.« Gesundheits- und Sozialpolitik(1/2012): S. $16-22$.

Björnberg, A. (2012): Euro Health Consumer Index. Health Consumer Powerhouse.

Dorresteijn, J. A., D. M. Kriegsman, et al. (2010): „Complex interventions for preventing diabetic foot ulceration. "Cochrane Database Syst Rev(1): CDoo7610.

Gensichen, J., C. Muth, et al. (2006): "[The future is chronic: German primary care and the Chronic Care Model - The comprehensive principles in the proactive treatment of the chronically ill]. « Z Arztl Fortbild Qualitatssich 100(5): 365-374.

Gersch, M. (2011): "Quo Vadis IV? - Monitoring Integrierte Versorgung. " Deutsche Gesellschaft für Integrierte Versorgung, mimeo Berlin.

Grobe, T. G., H. Dörning, et al. (2003): Auswertung der GEK-Gesundheitsberichterstattung, Schwerpunkt: Charakterisierung von Hochnutzern im Gesundheitssystem - präventive Potenziale? GEK-Gesundheitsreport. G. Ersatzkasse. Schwäbisch Gmünd, Asgard.

Henze, M. and S. J. Pöppl (2005): "Integrierte Versorgung durch Kooperation der sektoralen Gesundheitssysteme." Telemedizinführer: S. 225-229.

Inglis, S. C., R. A. Clark, et al. (2010): "Structured telephone support or telemonitoring programmes for patients with chronic heart failure."Cochrane Database Syst $\operatorname{Rev}(8)$ : CDo07228.

Jahn, E. (1975, S. 42): Systemanalyse der medizinischen Versorgung in der Bundesrepublik. Integrierte medizinische Versorgung: Notwendigkeiten-Möglichkeiten-Grenzen. WSI Forum am 25. und 26. April 1975. WSI Studie Nr. 32, 42. Bund Verlag Köln.

Lambrinou, E., F. Kalogirou, et al. (2012): „Effectiveness of heart failure management programmes with nurse-led discharge planning in reducing re-admissions: A systematic review and meta-analysis." International Journal of Nursing Studies 49(5): 610-624.

Lemmens, K. M., L. C. Lemmens, et al. (2011): "Chronic care management for patients with COPD: a critical review of available evidence." J Eval Clin Pract.
Mühlbacher, A., S. Lubs, et al. (2006): Status Quo der Integrierten Versorgung in Deutschland - eine empirische Analyse -. Zentrum für innovative Gesundheitstechnologie (ZiG). Berlin.

Niesink, A., J. C. Trappenburg, et al. (2007): "Systematic review of the effects of chronic disease management on quality-of-life in people with chronic obstructive pulmonary disease.« Respir Med 101(11): 2233-2239.

OECD (2011): Health at a Glance 2011: OECD Indicators. OECD Publishing. http://dx.doi. org/10.1787/health_glance-2011-en.

Ouwens, M., M. Hulscher, et al. (2009): »lmplementation of integrated care for patients with cancer: a systematic review of interventions and effects." Int J Qual Health Care 21(2) 137-144.

Peytremann-Bridevaux, I., P. Staeger, et al. (2008): "Effectiveness of chronic obstructive pulmonary disease-management programs: systematic review and meta-analysis.« Am J Med 121(5): 433-443 e434.

Phillips, C. O., R. M. Singa, et al. (2005): "Complexity of program and clinical outcomes of heart failure disease management incorporating specialist nurse-led heart failure clinics. A meta-regression analysis.« Eur J Heart Fail 7(3): 333-341.

Schmacke, N. (2011): "Qualitätsmanagement im Gesundheitswesen: der weite Weg der Evidenzbasierung in der Versorgungsforschung." Gesundheits- und Sozialpolitik(3/11): 32-36.

Seitz, P., T. Rosemann, et al. (2011): »Interventions in primary care to improve cardiovascular risk factors and glycated haemoglobin (HbA1c) levels in patients with diabetes: a systematic review." Diabetes Obes Metab 13(6): 479-489.

Smith, S. M., S. Allwright, et al. (2007): »Effectiveness of shared care across the interface between primary and specialty care in chronic disease management. "Cochrane Database Syst Rev(3): CDoo4910.

SVR (2012): Sondergutachten: Wettbewerb an der Schnittstelle zwischen ambulanter und stationärer Gesundheitsversorgung, Nr. 209. Sachverständigenrat zur Begutachtung der Entwicklung im Gesundheitswesen.

SVR KAG (2003): Finanzierung, Nutzerorientierung und Qualität, Nr. 712. Gutachten 2003. Sachverständigenrat für die Konzertierte Aktion im Gesundheitswesen.

The Commonwealth Fund. (2006): „2006 International Health Policy Survey of Primary Care Physicians." Retrieved 17.09.2012, from http://www.commonwealthfund.org/ Surveys/2006/2006-International-Health-Policy-Survey-of-Primary-Care-Physicians.aspx. 